\section{Variable expressivity of ocular associations of foveal hypoplasia in a family}

A Vincent ${ }^{1}$, V Kemmanu ${ }^{2}$, R Shetty ${ }^{1}$, V Anandula ${ }^{3}$, B Madhavarao ${ }^{2}$ and B Shetty

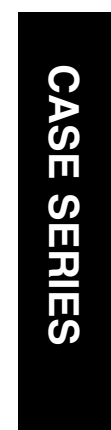

${ }^{1}$ Department of Electrophysiology and Medical Retina, Narayana Nethralaya, Superspeciality Eye Hospital and Post Graduate Institute of Ophthalmology, Bangalore, Karnataka, India

${ }^{2}$ Department of Pediatric Ophthalmology and Strabismus, Narayana Nethralaya, Superspeciality Eye Hospital and Post Graduate Institute of Ophthalmology, Bangalore, Karnataka, India

${ }^{3}$ Department of Ocular Genetics, Narayana Nethralaya, Superspeciality Eye Hospital and Post Graduate Institute of Ophthalmology, Bangalore, Karnataka, India

Correspondence: A Vincent, Department of

Electrophysiology and Medical Retina, Narayana Nethralaya, Superspeciality Eye Hospital and Post Graduate Institute of Ophthalmology, 121/C, Chord Road, Rajaji Nagar 1st 'R' Block,

Bangalore 560010,

Karnataka, India

Tel: + 9180 66121362;

Fax: + 918023377329

E-mail: ajoytvincent@

rediffmail.com angiogram (FFA) (Topcon TRC 50EX, Tokyo, Japan) showed the absence of foveal avascular zone (FAZ) in both eyes (Figure 3). A full-field electroretinogram (ERG) (Metrovision,

Pérenchies, France) showed normal responses in the left eye and reduced amplitude responses in the right eye. The subnormal ERG amplitude obtained from the right eye was attributed to
Received: 15 December 2008

Accepted in revised form:

11 June 2009

Published online: 10 July 2009

Financial disclosure: None 


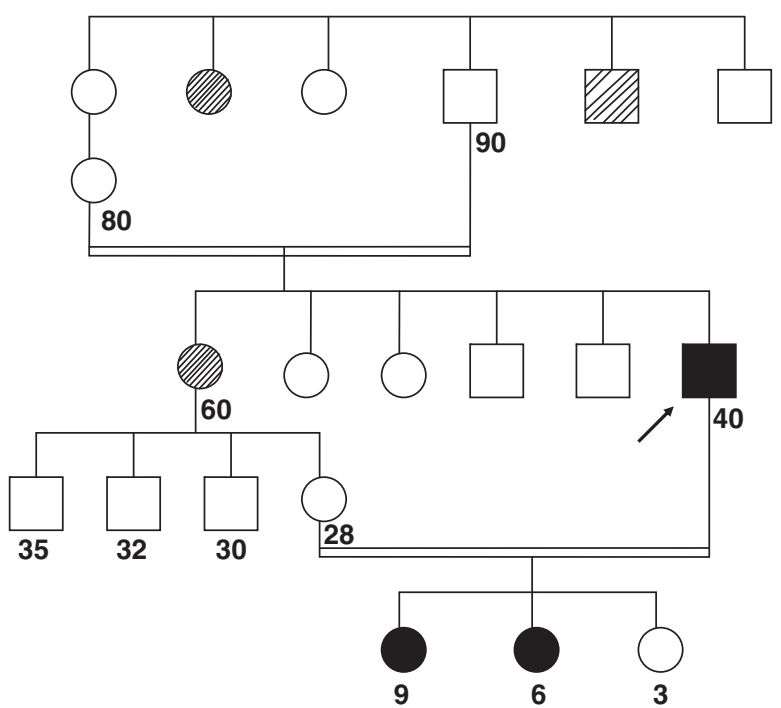

Figure 1 Pedigree charting of the family. Pedigree of a fivegeneration family associated with foveal hypoplasia showing autosomal recessive (pseudo dominant) inheritance. The Fully shaded symbol $(\rightarrow)$ indicates affected members who have been completely evaluated. The partially shaded symbol $(\rightarrow)$ indicates members who are affected by symptoms of esotropia, poor vision, and nystagmus. ERG, FFA, and OCT were not performed in these subjects.

the presence of uveal coloboma in that eye. A diagnosis of foveal hypoplasia with associated microphthalmos and right retinochoroidal coloboma was made.

\section{Case 2}

The 9-year-old daughter of case 1, with similar symptoms, was found to be a high hypermetrope, accepting a correction of $+8.5 \mathrm{DSph} /-2.00 \mathrm{DCyl} \times 160^{\circ}$ and $+7.50 \mathrm{DSph} /-1.25 \mathrm{DCyl} \times 20^{\circ}$ in the right and left eyes, respectively. The BCVA for distance and near was $6 / 30$ and N8, respectively, in both eyes. She had an alternating esotropia of $60 \mathrm{PD}$ with pendular nystagmus. Colour vision was normal. The horizontal corneal diameter was $11 \mathrm{~mm}$ in both eyes. The TAL was 19.49 and $19.53 \mathrm{~mm}$ in the right and left eyes, respectively. The fundus was unremarkable except for absent foveal reflexes in both eyes (Figure 2). FFA confirmed the absence of FAZ (Figure 3). Optical coherence tomography (OCT) (Ophthalmic Technologies Inc., Ontario, Canada) showed the absence of foveal pits in both eyes, and the ERG was normal. A diagnosis of foveal hypoplasia with associated microphthalmos was made.

\section{Case 3}

The 6-year-old younger daughter of case 1 also had similar complaints. She accepted a correction of
$+7.0 \mathrm{DSph} /-2.75 \mathrm{DCyl} \times 10^{\circ}$ and $+6.5 \mathrm{DSph} /-2.0$ DCyl $\times 180^{\circ}$ in the right and left eyes, respectively. Her BCVA for distance and near was $6 / 30$ and N8, respectively, in both eyes. She had an alternating esotropia of $60 \mathrm{PD}$, bilateral grade 3 inferior oblique over action, and pendular nystagmus. Her colour vision was normal and the horizontal corneal diameter was $11 \mathrm{~mm}$ in both eyes. The TAL was 20.70 and $20.54 \mathrm{~mm}$ in the right and left eyes, respectively. The fundus was unremarkable except for an absent foveal reflex (Figure 2). The FFA confirmed the absence of FAZ (Figure 3), whereas the OCT showed absence of a foveal pit and the ERG was normal. A diagnosis of isolated foveal hypoplasia was made.

\section{Discussion}

Foveal hypoplasia is used clinically to describe maculae where the foveal pit is poorly demarcated, with absence of the FAZ implying the lack of centrally specialized structures needed for good visual acuity. OCT in foveal hypoplasia shows the lack of a foveal pit, which is replaced by the central lengthening of the cone's outer segments and widening of the outer nuclear layer in some cases. ${ }^{6}$ Fovea plana is a term recently suggested to denote only the anatomic lack of a foveal pit $^{6}$ with no functional implications.

Isolated foveal hypoplasia is suggested to have autosomal recessive (AR) inheritance ${ }^{4}$ and is the only common feature in all three cases. Foveal hypoplasia with or without ocular associations has also been described..$^{7-9}$ Microphthalmos by definition is an eye that has an axial length that is 2 SDs smaller than what is normally expected at that age. This equates to $19.2 \mathrm{~mm}$ at 1 year and $20.9 \mathrm{~mm}$ at adulthood. ${ }^{10,11}$ In this series, microphthalmos without the accompanying microcornea was present in two cases. ${ }^{10,12}$ The pedigree chart (Figure 1) shows AR inheritance. The highlight of this series is the variable expressivity of ocular associations in foveal hypoplasia in AR inheritance pattern in the same family, which has not been reported earlier. This suggests that AR foveal hypoplasia may express as an isolated entity or be associated with varied developmental ocular abnormalities. Although uveal coloboma is a known association in microphthalmos, ${ }^{13}$ this is the first report of chorioretinal coloboma as an association of foveal hypoplasia.

Foveal hypoplasia, microphthalmos, and retinochoroidal coloboma occur because of defects in embryogenesis at various stages of development of the fetus ${ }^{14}$ owing to defective gene expression. This is an interesting case series of an AR family with foveal hypoplasia. 


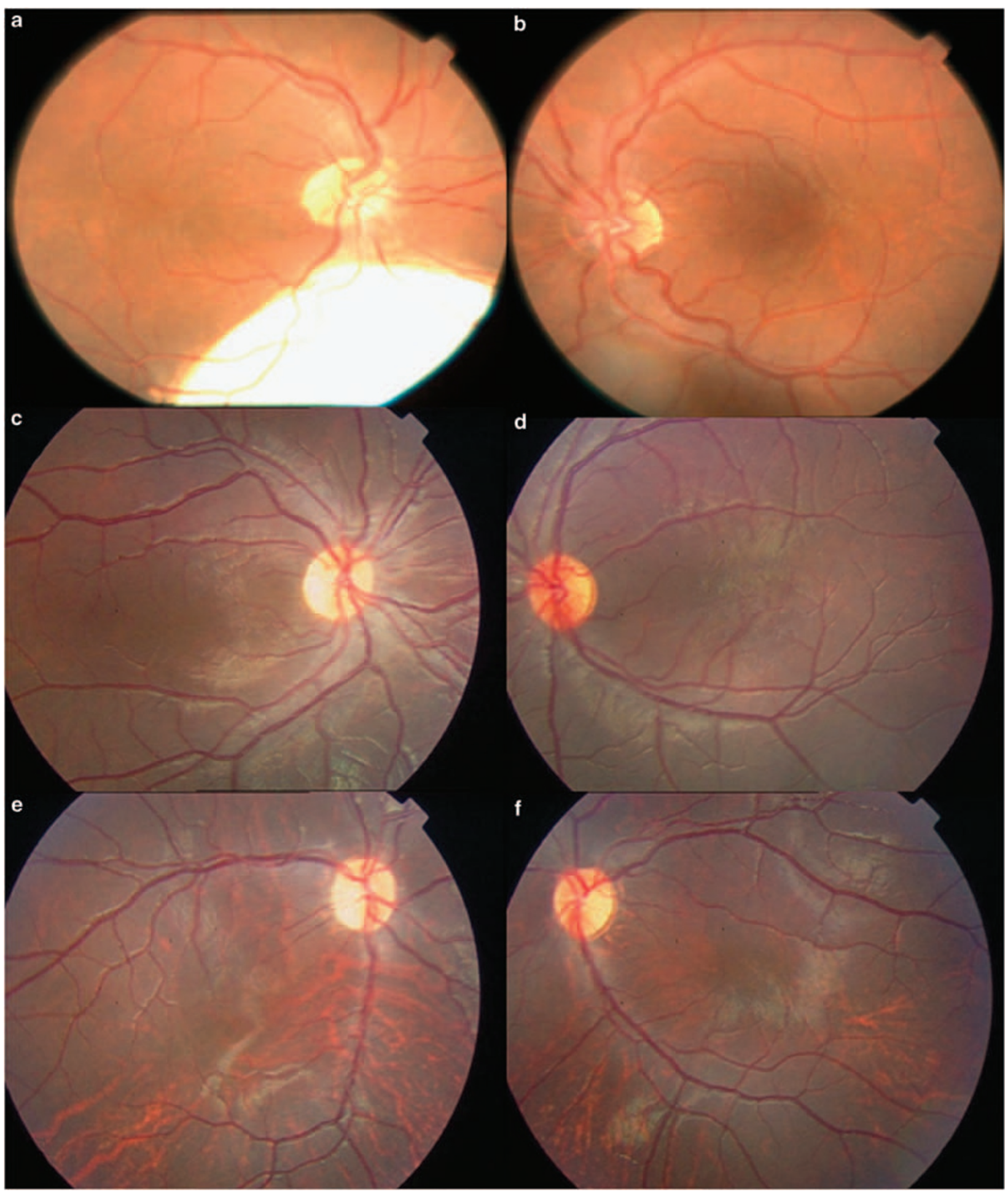

Figure 2 Fundus photographs of the three cases. (a) and (b) are the fundus photographs of the right and left eyes, respectively, of case 1. Note the poorly defined foveal zone in both eyes and the large typically inferior chorioretinal coloboma in the right eye. (c) and (d) are the fundus photographs of the right and left eyes, respectively, of case 2. The foveal zone is poorly defined in both eyes. (e) and (f) are the fundus photographs of the right and left eyes, respectively, of case 3 . In addition to the poorly developed foveal zone, also note the extortion of the fundus owing to the overaction of the inferior oblique muscle. 


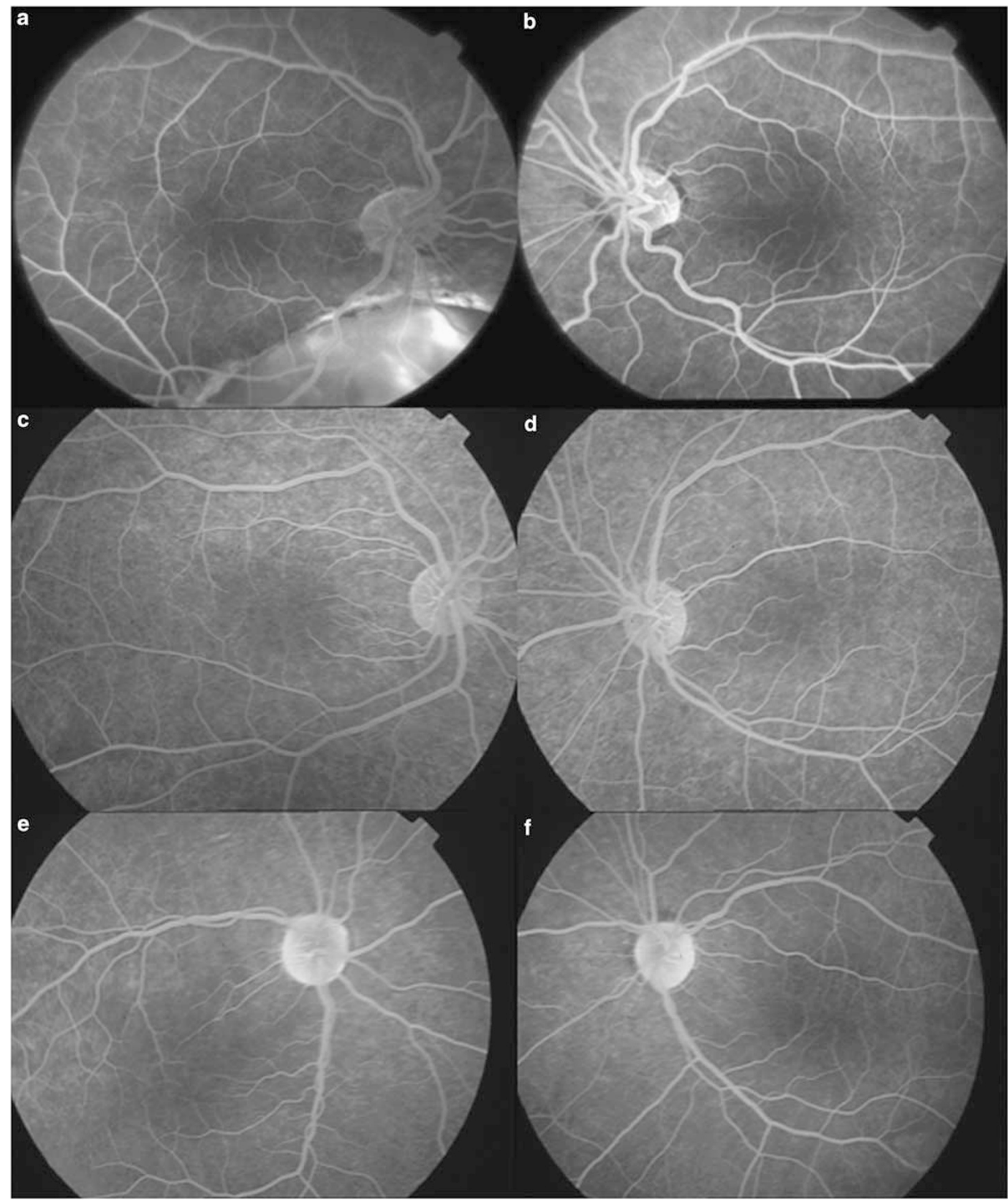

Figure 3 Fundus fluorescein angiogram (FFA) in the three cases showing the absence of foveal avascular zone (FAZ) in all of them. (a) and (b) are FFAs of the right and left eyes, respectively, of case 1. In addition to the absence of FAZ, note the large uveal coloboma in the right eye. (c) and (d) are FFAs of the right and left eyes, respectively, of case 2. (e) and (f) are FFAs of the right and left eyes, respectively, of case 3 . 


\section{References}

1 Francois J. Heredity in Ophthalmology. 1961; 153: 519.

2 Duke-Elder S. System of Ophthalmology. 1963; 3(2): 652-653.

3 Waardenburg PJ. Genetics and ophthalmology. 1963; 2: 1722-1723.

4 Curran RE, Robb RM. Isolated foveal hypoplasia. Arch Ophthalmol 1976; 94(1): 48-50.

5 O'Donnell Jr FF, Pappas HR. Autosomal dominant foveal hypoplasia and presenile cataracts. Arch Ophthalmol 1982; 100(2): 279-281.

6 Marmor MF, Choi SS, Zawadzki RJ, Werner JS. Visual insignificance of the foveal pit. Reassessment of foveal hypoplasia as fovea plana. Arch Ophthalmol 2008; 126(7): 907-913.

7 Azuma N, Nishina S, Yanagisawa H, Okuyama T, Yamada M. PAX6 missense mutation in isolated foveal hypoplasia. Nat Genet 1996; 13: 141-142.

8 Azuma N, Yamaguchi Y, Handa H, Hayakawa M, Kanai A, Yamada M. Missense mutation in the alternative splice region of the PAX6 gene in eye anomalies. Am J Hum Genet 1999; 65: 656-663.

9 Pal B, Mohamed MD, Keen TJ, Williams GA, Bradbury JA, Sheridan E et al. A new phenotype of recessively inherited foveal hypoplasia and anterior segment dysgenesis maps to a locus on chromosome 16q23.2-24.2. J Med Genet 2004; 41: 772-777.

10 Elder MJ. Aetiology of severe visual impairment and blindness in microphthalmos. Br J Ophthalmol 1994; 78: 332-334.

11 Weiss AH, Kouseff BG, Ross EA, Longbottom J. Simple microphthalmos. Arch Ophthalmol 1989; 107: 1625-1630.

12 Larsen JS. The sagittal growth of the eye. IV. Ultrasonic measurement of the axial length of the eye from birth to puberty. Acta Ophthalmol (Copenh) 1971; 49(6): 873-886.

13 Warburg M. Classification of microphthalmos and coloboma. J Med Genet 1993; 30: 664-669.

14 Taylor D, Hoyt CS. Pediatric Ophthalmology and Strabismus, 3rd ed. Elsevier: Philadelphia, PA, 2005. 